\title{
Calculation of the Spinning Speed of a Free Electron
}

\author{
Mesude Saglam', Burcin Bayram², Ziya Saglam³, Hanasli Gur4 \\ ${ }^{1}$ Department of Physics, Faculty of Science, University of Ankara, Ankara, Turkey \\ ${ }^{2}$ Department of Physics, Miami University, Oxford, OH, USA \\ ${ }^{3}$ Department of Physics, Faculty of Science, University of Aksaray, Aksaray, Turkey \\ ${ }^{4}$ Department of Engineering in Physics, Ankara University, Ankara, Turkey \\ Email: saglam@science.ankara.edu.tr
}

How to cite this paper: Saglam, M., Bayram, B., Saglam, Z. and Gur, H. (2020) Calculation of the Spinning Speed of a Free Electron. Journal of Modern Physics, 11, 9-15.

https://doi.org/10.4236/jmp.2020.111002

Received: November 2, 2019

Accepted: January 5, 2020

Published: January 8, 2020

Copyright $\odot 2020$ by author(s) and Scientific Research Publishing Inc. This work is licensed under the Creative Commons Attribution International License (CC BY 4.0).

http://creativecommons.org/licenses/by/4.0/

\begin{abstract}
In a recent work, we calculated the magnetic field inside a free electron due to its spin, and found it to be about $B=8.3 \times 10^{13} \mathrm{~T}$. In the present study we calculate the spinning speed of a free electron in the current loop model. We show that spinning speed is equal to the speed of light. Therefore it is shown that if electron was not spinning the mass of electron would be zero. But since spinning is an unseparable part of an electron, we say that mass of electron is non-zero and is equal to $\left(m=9.11 \times 10^{-28} \mathrm{~g}\right)$.
\end{abstract}

\section{Keywords}

Spinning Speed, Intrinsic Current, Intrinsic Magnetic Field, The Intrinsic Flux of Electron, Current Loop Model

\section{Introduction}

Recently we have calculated the magnetic field inside a free electron due to spinning motion [1] and showed that it is about $8.3 \times 10^{13} \mathrm{~T}$. This field is about $8.3 \times$ $10^{11}$ times bigger than the highest magnetic field obtained in today's laboratories [2] [3] and $10^{3}$ times bigger than that in neutron stars (magnetars) [4] [5]. In that calculations [1], which are based on the current loop model, the intrinsic magnetic flux associated with its spinning motion of the electron which is calculated either by a semiclassical methode or by a full quantum mechanical solution of Dirac equation [6] [7] [8] gives the same result: $\Phi_{e}^{(s)}= \pm h c / 2 e$. The current loop model is mainly based on the magnetic top model which was first introduced by Barut et al. [9] and used by N. Rosen [10] and L. Schulman [11]. But the magnetic top model was rather primitive as the spin vector was only attached 
to that spherical charge distribution. To overcome this difficulty we introduced current loop model [6]. In this study using the current loop model we have found that: electron's spinning angular frequency, $\omega_{s}=7.77 \times 10^{22} \mathrm{rad} / \mathrm{sec}$. Most importantly through this model we had calculated magnetic flux associated with its spinning motion and we have found the above mentioned result: $\Phi_{e}^{(s)}= \pm h c / 2 e= \pm \Phi_{0} / 2$ where (+) sign stands for spin-down electron and (-) sign for spin-up electron. Therefore the current loop model is the best one to describe the magnetic properties of the electron. Further we have found that the spinning speed of an electron is exactly equal to speed of light. Furthermore, if $v$ $=c$, according to the relativity theory [12], the relativistic mass, $m$ of a speedy particle will have a non-zero limit if and only if $m_{0}$ is zero:

$$
m=\frac{m_{0}}{\sqrt{1-\frac{v^{2}}{c^{2}}}}
$$

where $m_{0}$ is the mass with zero speed. Therefore the Equation (1) can only be non-zero if and only if $m_{0}$ is zero. Since spinning is an unseperable part of electron we may say that mass of electron is non-zero and is equal to $9.11 \times$ $10^{-28} \mathrm{~g}$.

\section{Formalism}

As we said earlier the current loop model [6] is an idealistic model for a spining electron. In this model the spining electron is made equivalent to a circular current loop with the radius $R$ in $x$ - $y$ plane and the electron motion is considered in two parts namely an "external" motion which can be interpreted as the motion of the center of mass (and hence the central of charge) and an "internal" one whose average disappears in the calssical limit. The latter is caused by the spin of the electron.

To calculate the quantum flux for any quantum orbit [6] we calculate the magnetic flux for one turn, then multiply it by the number of turns, during the cyclotron period $T_{\dot{c}}$

$$
\Phi=\int \boldsymbol{B} \cdot \mathrm{d} \boldsymbol{a}=\int_{0}^{T_{c}} \frac{\boldsymbol{B}}{2} \cdot\left(\boldsymbol{r} \times \frac{\mathrm{d} \boldsymbol{r}}{\mathrm{d} t}\right) \mathrm{d} t=\frac{\omega_{s}}{\omega_{c}} \int_{0}^{T_{s}} \frac{\boldsymbol{B}}{2} \cdot\left(\boldsymbol{r} \times \frac{\mathrm{d} \boldsymbol{r}}{\mathrm{d} t}\right) \mathrm{d} t=\frac{\omega_{s}}{\omega_{c}} \pi R^{2} B
$$

Here we distinguish spin angular frequency $\omega_{s}$ from the cyclotron frequency $\omega_{c}=e B / m c$. When an electron is placed in an external magnetic field $B$, during the cyclotron period $T_{c}$ it completes one turn around the cyclotron orbit, but it spins $\left(\omega_{\mathrm{s}}\right)$ times about itself (Figure 1) [13]. We will see that $\left(\omega_{\mathrm{s}} \gg \omega_{c}\right)$.

Now we want to look at the Equation (2) in detail: To consider the spin dependence in the flux expression [6], we assume that the spin angular momentum of electron is produced by the fictitious point charge $(-e)$ rotating in a circular orbit with a radius $R$ in $x-y$ plane and an angular frequency, $\omega_{s}$; that is what we call the current loop model. In the presence of a magnetic field, $\boldsymbol{B}=B \hat{z} \quad(B>$ 0 ), the vector going to this fictitious point charge can be written as: 


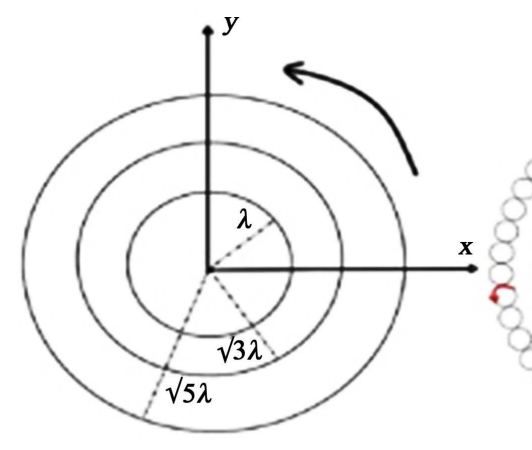

(a)

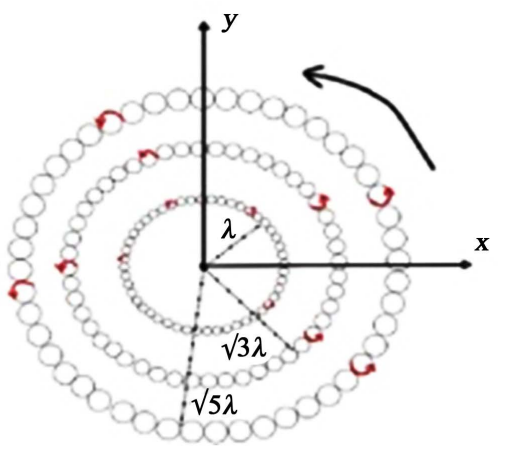

(b)

Figure 1. Landau orbits for electrons: (a) without spin; (b) in real space for spin-up electrons.

$$
\dot{r}=\boldsymbol{r}+\boldsymbol{R}
$$

where $\boldsymbol{r}$ is the vector going from origin to the centre of mass of the electron and $\boldsymbol{R}$ is the vector going from centre of mass to this fictitious point charge $(R \ll r)$. So the vector $\boldsymbol{r}$ in Equation (3) reads:

$$
\boldsymbol{r}=r \cos \left(\omega_{c} t+\vartheta_{c}\right) \hat{x}+r \sin \left(\omega_{c} t+\vartheta_{c}\right) \hat{y}
$$

here $\vartheta_{c}$ is the angle at $t=0$.

Depending on the spin orientation, the vectors $R(\uparrow)$ and $R(\downarrow)$ namely for spin up and spin down electrons read:

$$
\begin{aligned}
& \boldsymbol{R}(\uparrow)=R \cos \left(\omega_{s} t+\vartheta_{s}\right) \hat{x}-R \sin \left(\omega_{s} t+\vartheta_{s}\right) \hat{y} \\
& \boldsymbol{R}(\downarrow)=R \cos \left(\omega_{s} t+\vartheta_{s}\right) \hat{x}+R \sin \left(\omega_{s} t+\vartheta_{s}\right) \hat{y}
\end{aligned}
$$

where $\vartheta_{s}$ is the angle at $t=0$.

Here we distinguish the spin angular frequency $\omega_{s}$ from the cyclotron angular frequency $\omega_{c}=e B / m c$. During the cyclotron period, $T_{c}$ electron completes one turn around the cyclotron orbit, but it spins $\omega_{s} / \omega_{c}$ times about itself and hence the fictitious point charge completes $\omega_{s} / \omega_{c}$ loops with the area $\pi R^{2}$. It can be shown that the number of turns, $N=\omega_{s} / \omega_{c}$ is very large. We will see that $\omega_{s}=7.77 \times 10^{22} \mathrm{rad} / \mathrm{sec}$ and $\omega_{c}=e B / m c$ can be made as small as possible. But if we take a huge magnetic field let us say for $B=10, T=10^{5} \mathrm{G}$ then the value of $\omega_{c}$ becomes: $\omega_{c} \cong 1.76 \times 10^{12} \mathrm{rad} / \mathrm{sec}$. Therefore we can say $\omega_{s} \gg \omega_{c}$.

If we take the time derivative of (3), we find the corresponding velocities:

$$
\dot{v}=v+V
$$

Here $\boldsymbol{v}$ and $\boldsymbol{v}$ are the velocities of the electron itself and fictitious point charge $(-e)$ with respect to the origin and $V$ is the velocity of the fictitious point charge with respect to the centre of mass of the electron.

Next, following Saglamand Boyacioglu [6], we can calculate the total magnetic flux $\Phi^{\prime}(\uparrow)$ contained within the spinning orbit of the fictitious charge during 
the time interval $T_{c}$ :

$$
\begin{aligned}
\Phi^{\prime}(\uparrow) & =\oint \boldsymbol{B} \cdot \frac{\boldsymbol{r} \times \mathrm{d} \boldsymbol{r}}{2}=\oint_{0}^{T_{c}} \frac{\boldsymbol{B}}{2} \cdot\left(\boldsymbol{r}^{\prime} \times \boldsymbol{v}^{\prime}\right) \mathrm{d} t \\
& =\oint_{0}^{T_{c}} B\left[\omega_{c} r^{2}-\omega_{s} R^{2}+\text { cross terms }\right] \mathrm{d} t
\end{aligned}
$$

where the cross terms contains the product of different angular frequencies like $\cos \omega_{c} t \cos \omega_{s} t \cdots$ and so on. When we take the integral of cross terms, they vanish and the Equation (8) reduces to

$$
\Phi^{\prime}(\uparrow)=\frac{B}{2}\left[\omega_{c} r^{2}-\omega_{s} R^{2}\right] \frac{2 \pi}{\omega_{c}}=\pi r^{2} B-\frac{\omega_{s}}{\omega_{c}} \pi R^{2} B
$$

As it is shown in [6] that the first term can be written as:

$$
\pi r^{2} B=\left(n+\frac{1}{2}\right) \Phi_{0}=\left(n+\frac{1}{2}\right) \frac{h c}{e}
$$

which is the quantum flux without considering electron spin. But the second term in Equation (9) is the contribution of spin to the total flux for spin-up electron. To calculate it we use Equation (20) of the following section:

$$
\omega_{s}=\frac{\hbar}{m R^{2}} \equiv \frac{h}{2 \pi m R^{2}}
$$

Substituting Equation (10) and Equation (11) in Equation (9) and using the relation $\omega_{c}=e B / m c$ we find:

$$
\Phi^{\prime}(\uparrow)=\left(n+\frac{1}{2}\right) \frac{h c}{e}-\frac{h c}{2 e}=\frac{n h c}{e}=n \Phi_{0} \quad(n=0,1,2,3, \cdots)
$$

If we follow a similar procedure for spin-down electron the total flux for spin-down electron takes the form:

$$
\Phi^{\prime}(\downarrow)=\pi r^{2} B+\frac{\omega_{s}}{\omega_{c}} \pi R^{2} B
$$

With a similar procedure we find

$$
\Phi^{\prime}(\downarrow)=\left(n+\frac{1}{2}\right) \frac{h c}{e}+\frac{h c}{2 e}=(n+1) \frac{h c}{e}=(n+1) \Phi_{0} \quad(n=0,1,2,3, \cdots)
$$

From Equations (12) and (14) it is seen that the spin contribution to the total flux is $-\Phi_{0} / 2$ for spin-up electron and $\Phi_{0} / 2$ for spin-down electron:

$$
\begin{gathered}
\Phi(\uparrow)=-\frac{h c}{2 e}=-\frac{\Phi_{0}}{2} \\
\Phi(\downarrow)=\frac{h c}{2 e}=\frac{\Phi_{0}}{2}
\end{gathered}
$$

Here the obtained net results show that the current loop model for electron spin is realy a satisfactory model.

To proceed further we write the spin magnetic moment $\mu$ for a free electron [14]:

$$
\boldsymbol{\mu}=-g \mu_{B} S
$$

Here $\hbar \boldsymbol{S}$ the spin angular momentum of the electron. 
When we introduce the magnetic field $\boldsymbol{B}=B \hat{z}$, the $z$-component of the magnetic moment for a spin-down electron [6] becomes ( $g=2$ for a free electron);

$$
\mu_{z}=\frac{e \hbar}{2 m c}
$$

In the current loop model $z$-component of the magnetic moment for a spin-down electron is:

$$
\left(\mu_{e}\right)_{z}=\frac{I A}{c}=\frac{e R^{2} \omega_{s}}{2 c}
$$

where $A=\pi R^{2}$ and $R$ is the radius of the current loop. From Equation (17) and (18) we obtain:

$$
R=\left(\frac{\hbar}{m \omega_{s}}\right)^{1 / 2}
$$

If we solve $\omega_{s}$, from Equation (19) we find the spinning angular velocity of electron in terms of the radius of the current loop, $R$ :

$$
\omega_{s}=\frac{\hbar}{m R^{2}}
$$

Now with a rough approach we want to calculate spinning speed of electron in the current loop model. If we take the radius of this current loop equal to the electron radius, $R(e l)=2.82 \times 10^{-13} \mathrm{~cm}$ and the mass of electron, $m=9.11 \times 10^{-28} \mathrm{~g}$ [15], from Equation (20) we find:

$$
\omega_{s}^{\prime}=9.11 \times 10^{25} \mathrm{rad} / \mathrm{sec}
$$

and writing $v(e l)=R(e l) \omega_{s}^{\prime}$ we find electron velocity as:

$$
v(e l)=410 \times 10^{10} \mathrm{~cm} / \mathrm{sec}
$$

which is larger that the speed of light, $c$. This is imposible! The reason for this is that the radius of the mentioned current loop is not equal to the electron radius. Therefore the right value of the radius of this current loop must be taken in to account. We know that (please see the discussion section of [6]) the radius of the current loop is a dummy variable. As far as the flux calculations are concerned the radius $R$ of the current loop is phenomenal concept whose detailed calculation is not important. Therefore we chose the radius of this current loop such that the above speed should not exceed the speed of light, $c$.

$$
v=R \omega_{s}=c
$$

When we solve Equations (20) and (23) together we find:

$$
R=3.86 \times 10^{-11} \mathrm{~cm}
$$

and

$$
\omega_{s}=7.77 \times 10^{22} \mathrm{rad} / \mathrm{sec}
$$

So we can say that in the current loop model electron is spinning in a circular ring of radius $R=3.86 \times 10^{-11} \mathrm{~cm}$ with the speed of light, $c$ and with an angular 
velocity $\omega_{s}=7.77 \times 10^{22} \mathrm{rad} / \mathrm{sec}$. Furthermore, since $v=c$, according to the relativity theory [12], the relativistic mass, $m$ of a speedy particle will have a non-zero limit if and only if $m_{0}$ is zero:

$$
m=\frac{m_{0}}{\sqrt{1-\frac{v^{2}}{c^{2}}}}
$$

That is to say; If the spinning speed is equal to the speed of light, $c$, the Equation (26) can only be non-zero if and only if $m_{0}$ is zero. Since spinning is an unseperable part of electron we may say that mass of electron is non-zero and is equal to the mass, $m=9.11 \times 10^{-28} \mathrm{~g}$.

\section{Conclusions}

We have calculated the spinning speed of a free electron in the current loop model which is a correct one as it produced the magneticflux due to spin of electron as $\Phi_{e}^{(s)}=\frac{h c}{2 e}=\Phi_{0} / 2$.

By using the Equation (20) and $R \omega_{s}=c$, we were able to calculate the radius of this current loop $R$ and cyclotron frequency, $\omega_{s}$ of electron on this current loop. These values are: $R=3.86 \times 10^{-11} \mathrm{~cm}$ and $\omega_{\mathrm{s}}=7.77 \times 10^{22} \mathrm{rad} / \mathrm{sec}$.

More importantly it is shown that if electron was not spinning the mass of electron would be zero. But since spinning is unseparable part of electron we say that mass of electron is non-zero and is equal to $m=9.11 \times 10^{-28} \mathrm{~g}$.

\section{Conflicts of Interest}

The authors declare no conflicts of interest regarding the publication of this paper.

\section{References}

[1] Saglam, M., Sahin, G. and Gur, H. (2018) Results in Physics, 10, 973. https://doi.org/10.1016/j.rinp.2018.08.014

[2] http://phys.org/news/2016-11-national-maglab-racks-world-hybrid.html

[3] https://www.ru.nl/hfml/facility/experimental/magnets

[4] Potekhin, A.Y., Yakovlev, D.G., Chabrier, G. and Gnedin, O.Y. (2003) The Astrophysical Journal, 594, 404-418. https://doi.org/10.1086/376900

[5] Alaa, I.I., Swank, J.H. and William, P. (2003) The Astrophysical Journal, 584, L17-L21. https://doi.org/10.1086/345774

[6] Saglam, M. and Boyacioglu, B. (2002) International Journal of Modern Physics B, 16, 607. https://doi.org/10.1142/S0217979202010038

[7] Wan, K. and Saglam, M. (2006) International Journal of Theoretical Physics, 45, 1132. https://doi.org/10.1007/s10773-006-9118-z

[8] Yilmaz, O., Saglam, M. and Aydin, Z.Z. (2007) Old and New Concepts of Physics, 4, 141. https://doi.org/10.2478/v10005-007-0007-x

[9] Barut, A.O., Bozic, M. and Maric, Z. (1992) Annals of Physics, 214, 53. https://doi.org/10.1016/0003-4916(92)90061-P 
[10] Rosen, N. (1951) Physical Review, 82, 621. https://doi.org/10.1103/PhysRev.82.621

[11] Schulman, L. (1968) Physical Review, 176, 1558. https://doi.org/10.1103/PhysRev.176.1558

[12] Griffiths, D.J. (1999) Introduction to Electrodynamics. 3rd Edition, Prentice-Hall, London.

[13] Saglam, Z. and Boyacioglu, B. (2018) Acta Physica Polonica A, 133, 1129-1132. https://doi.org/10.12693/APhysPolA.133.1129

[14] Sakurai, J.J. and Napolitano, J. (2010) Modern Quantum Mechanics. 2nd Edition, Pearson Education Inc., London.

[15] Feynman, R.P. and Leighton, R.B. (1964) Matthew Sands. 4th Edition, Addison Wesley Publishing Company, Boston. 